\title{
LA-UR-13-29342
}

Approved for public release; distribution is unlimited.

Title: $\quad$ Final Report for NFE-13-0019: Analysis of Muon Radiography of the Toshiba Nuclear Critical Assembly Reactor and Path Forward

Author(s): $\quad$ Morris, Christopher

Intended for: $\quad$ Report

Issued: $\quad$ 2013-12-10

Disclaimer:

Los Alamos National Laboratory, an affirmative action/equal opportunity employer,is operated by the Los Alamos National

Security, LLC for the National NuclearSecurity Administration of the U.S. Department of Energy under contract DE-AC52-06NA25396.

By approving this article, the publisher recognizes that the U.S. Government retains nonexclusive, royalty-free license to publish or reproduce the published form of this contribution, or to allow others to do so, for U.S. Government purposes.

Los Alamos National Laboratory requests that the publisher identify this article as work performed under the auspices of the

U.S. Departmentof Energy. Los Alamos National Laboratory strongly supports academic freedom and a researcher's right to publish; as an institution, however, the Laboratory does not endorse the viewpoint of a publication or guarantee its technical correctness. 


\section{Final Report for NFE-13-0019: Analysis of Muon Radiography of the Toshiba Nuclear Critical Assembly Reactor and Path Forward}

Spokesperson: C. L. Morris ${ }^{1}$

Participants: Jeffery Bacon, ${ }^{1}$ Yuichiro Ban, ${ }^{2}$ Konstantin Borozdin, ${ }^{1}$ J. M. Fabritius, ${ }^{1}$ Mikio Izumi, ${ }^{2}$ Haruo Miyadera, ${ }^{1,2}$ Shinya Mizokami, ${ }^{4}$ Yasuyuki Otsuka, ${ }^{4}$ John Perry, ${ }^{1}$ John Ramsey, ${ }^{1}$ Yuji Sano, ${ }^{2}$ Tsukasa Sugita, ${ }^{3}$ Daichi Yamada, ${ }^{4}$ Noriyuki Yoshida, ${ }^{2}$ and Kenichi Yoshioka ${ }^{3}$

${ }^{1}$ Los Alamos National Laboratory, Los Alamos, NM USA 87545

${ }^{2}$ Toshiba Corporation, 8 Shinsugita-cho, Isogo-ku, Yokohama, 235-8523, Japan

${ }^{3}$ Toshiba Corporation, 4-1 Ukishima-cho, Kawasaki-ku, Kawasaki, 210-0862, Japan

${ }^{4}$ Tokyo Electric Power Company, 1-1-3 Uchisaiwai-cho, Chiyoda-ku, Tokyo, Japan

A $1.2 \times 1.2 \mathrm{~m}^{2}$ muon tracker was moved from Los Alamos to the Toshiba facility at Kawasaki, Japan where it was used to take $\sim 4$ weeks of data radiographing the Toshiba Critical Assembly Reactor with cosmic ray muons. In this paper we describe the analysis procedure, show results of this experiment and compare the results to Monte Carlo predictions. The results validate the concept of using cosmic rays to image the damaged cores of the Fukushima Daiichi reactors.

\section{Introduction}

Cosmic ray muon radiograph has been proposed as a method for obtaining information about the location of the fissile material in the melted cores of the Fukushima Daiichi reactors. In a recent paper[1] the simulation code GEANT4[2] was used to track cosmic rays through a model of a boiling water reactor similar to Fukushima Daiichi Reactor number 1. [see the attached papers] The model of the reactor included all major structures, the reactor building, containment vessel and the pressure vessel. Calculations were performed for an intact core, a core with a $1 \mathrm{~m}$ diameter of material removed from the core and placed in the bottom of the pressure vessel, and with no core. The goal of the work was to compare images obtained using the stopping of cosmic rays with images made using the scattering of transmitted cosmic rays. 
Although muons of sufficient energy can be transmitted through thick objects, they continuously interact with the electrons and nuclei in the matter. These interactions consist of the Coulomb and the weak interaction between the muons and the electrons and nuclei. To a good approximation these can be treated separately and each can be used for radiography.[3] In the context of radiography, the weak interaction can be ignored.

The Coulomb interaction between muons the much lighter electrons results in continuous energy loss [4] and eventual stopping of the muons as they move through matter. In contrast the Coulomb interaction between muons and atomic nuclei results in deflections of the muon trajectory, but negligible energy changes. Because the integral of the Coulomb cross section is infinite, the solution to the problem of charged particle transport in matter is non-trivial. The number of collisions is large and this process results in a continuous increase of the angular divergence of the beam described by Coulomb multiple scattering.[5-7]

The stopping rate, $\frac{d N}{d x}$, of cosmic rays in material can be related to the energy spectrum, $\frac{d N(E=0)}{d E}$, as:

$$
\begin{aligned}
& \frac{d N}{d x}=\frac{d N}{d E} \frac{d E}{d x} \\
& \frac{d E}{d x}=K \frac{Z}{A} \frac{1}{\beta^{2}}\left[\frac{1}{2} \ln \left(\frac{2 m_{e} c^{2} \beta^{2} \gamma^{2} T_{\max }}{I^{2}}-\beta^{2}-\frac{\delta(\beta \gamma)}{2}\right]^{\prime}\right.
\end{aligned}
$$

Where $\mathrm{Z}$ and $\mathrm{A}$ are the atomic number and mass, $\beta=v / c$ where $\beta$ is the muon velocity and $\mathrm{c}$ is the velocity of light and $\gamma=\sqrt{\frac{1}{1-\beta^{2}}}, m_{e}$ is the electron mass, $l$ is the mean ionization potential, and $\delta$ is a function described in [8]. The low energy part of the spectrum depends upon altitude, azimuth, and overburden, and is best determined experimentally.

The multiple scattering is described by: 


$$
\begin{aligned}
& \frac{d N}{d \theta}=\frac{1}{2 \pi \theta_{0}^{2}} e^{-\frac{\theta^{2}}{2 \theta_{0}^{2}}} d \Omega \\
& \theta_{0}=\frac{14.1}{p \beta} \sqrt{\frac{L}{X_{0}}} \\
& \frac{1}{X_{0}}=\frac{K}{A}\left\{Z^{2}\left[L_{r a d}-f(Z)\right]+Z L_{r a d}^{\prime}\right\}
\end{aligned}
$$

where $d N / d \theta$ is the polar angular ( $\theta$ ) distribution of muons of momentum $p$ (in $\mathrm{MeV} / \mathrm{c}$ ) that pass through a distance $L$ of material. $K$ and $L_{\text {rad, }} L^{\prime}$ rad , and $f(Z)$ are described in reference [8]. The $Z^{2}$ dependence of radiation length, $X_{0}$, leads to a high sensitivity of Coulomb multiple scattering to high $Z$ materials.

The conclusions of Reference [1] are: the large Z-dependence of multiple scattering radiography makes it far better suited for reactor radiography when compared to stopping radiography; and that several weeks of exposure provide enough information to provide clear images of the core with $<1 \%$ mass sensitivity. These conclusions are apparent from the radiographs shown in Reference [1], where scattering and transmission radiography are compared. Although in four days the details of the core are clear in scattering radiography, little information is available from transmission radiography even after 6 weeks of exposure.

The simulations in Reference [1] assumed $50 \mathrm{~m}^{2}$ of detector on opposite sides of the reactor. The residual radiation on the site is still $\sim 1 \mathrm{mSv}$ in the locations where the detectors were placed in the simulation. Shielding is necessary to mitigate the detector backgrounds from $\gamma$-rays. The engineering and construction problems were partially addressed in further Monte Carlo studies where the detector locations and sizes were adjusted to fit in practical locations.[9] In addition, a new reconstruction technique that increases the sensitivity for commercial reactor geometries where the scattering of outgoing muons in the shielding and concrete build walls reduces the sensitivity of scattering radiography, displacement radiography, was described.

Finally, muon radiography of a small research reactor at the University of New Mexico, an AGN-201M, was demonstrated using the Los Alamos Mini Muon Tracker (MMT).[10] The University of New Mexico Research Reactor consists of $10.9 \mathrm{~kg}$ of polyethylene loaded with about $3.3 \mathrm{~kg}$ of enriched uranium. Moderator and shielding consisting of graphite, lead, water, and concrete surround the core. Even though the density of uranium in the core is only about $0.3 \mathrm{~g} / \mathrm{cm}^{3}$ the combination of simulation and data demonstrated sufficient sensitivity to detect the Uranium through the $10 \mathrm{~cm}$ thick lead reflector and water and concrete shielding surrounding the core. However, the sensitivity was not large. Here we present the results of a similar experiment aimed at measuring of the Toshiba Nuclear Critical Assembly (NCA) reactor, where the core although only $\sim 1 / 3$ scale is more similar to a commercial power reactor. 


\section{Configuration}

This experiment used the MMT detectors mounted outside of the reactor water vessel. A $0.5 \mathrm{~cm}$ thick steel plate and a $1 \mathrm{~cm}$ thick aluminum plate covered the front of the lower detector and some $28 \times 20 \times 12$ $\mathrm{cm}^{3}$ steel blocks were placed in front and behind $20 \times 20 \times 20$ concrete blocks in front of the upper detector to test the ability of muon tomography to view through overburden. The core was loaded with a configuration of 1.5 meter long $1 \mathrm{~cm}$ diameter $\mathrm{UO}_{2}$ ceramic fuel rods, on $1.5 \mathrm{~cm}$ centers, configured in a $40 \mathrm{~cm}$ diameter cylinder with a $20 \mathrm{~cm}$ void at its center. In addition, $3 \times 3$ assemblies of rods were placed to the sides in front and behind the cylinder as shown in Figure 2.

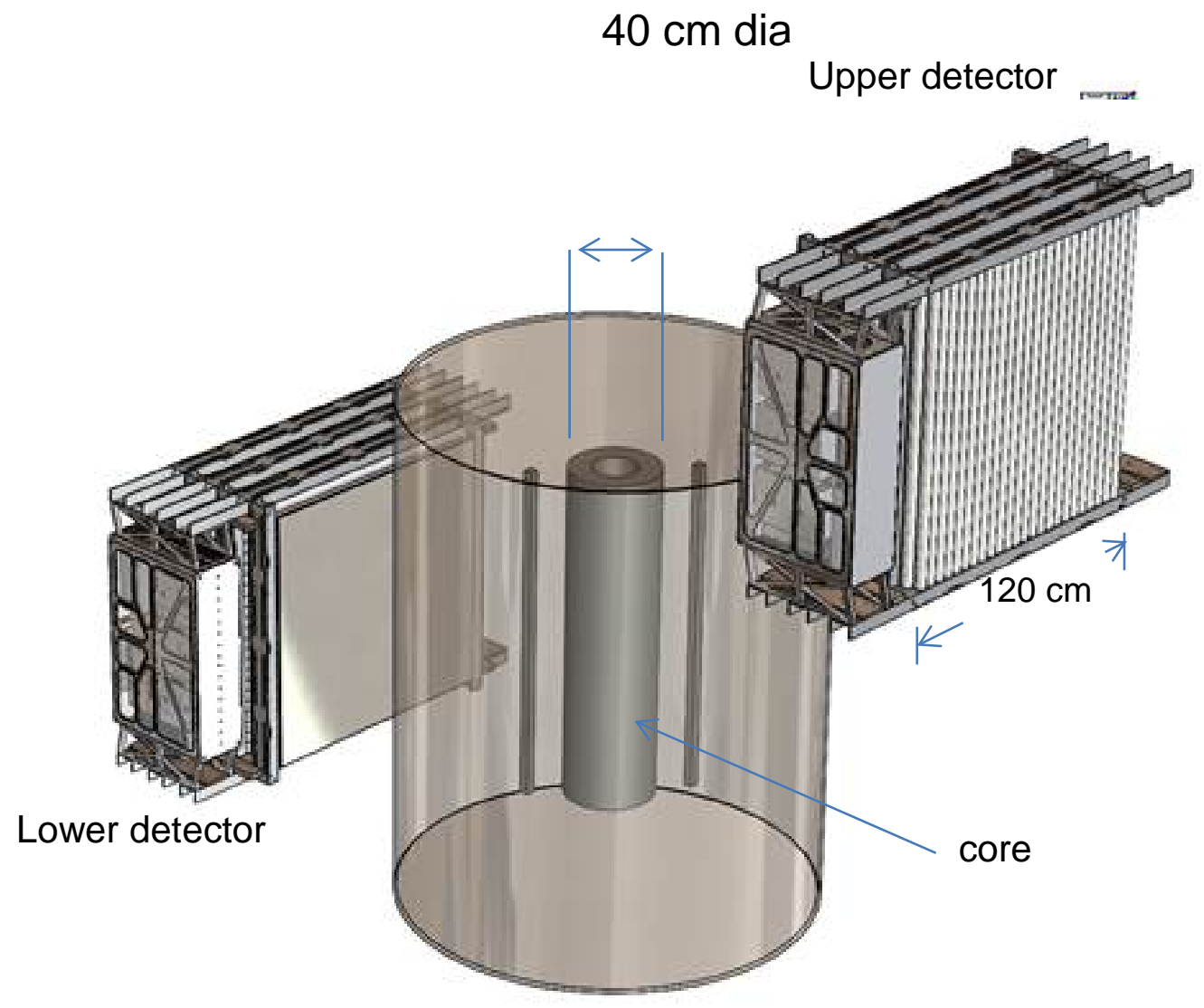

Figure 1) Layout for current measurements.

This geometry provided multiple objects to image: the center cylinder, the smaller fuel rod bundles to the sides and the steel blocks near the upper detectors. In order to accomplish this we have used the tomographic muon imaging techniques described in [11, 12].

\section{Results}


Approximately 4 weeks of data were taken in the configuration shown in Figure 2. In previous work it has been shown the lowest variance can be obtained from multiple scattering tomography by using a multi-energy group fit to the angular-distribution data to form an image of radiation lengths.[12] We have used that method here, and have calibrated it using the known path lengths through the NCA reactor. In Figure 3 we show the normalized angular distribution for some of the data through a region outside of the core, through the thickest part of the core and through the center of the core. Here we have fitted a function that includes an angular resolution, $9.1 \mathrm{mrad}$, and a fixed overburden, 6.4 Radiation Lengths (RL). These values are larger than in the typical vertical configuration of the tracker. Because they have little impact on the results, they have been treated as empirical parameters in this analysis. We attribute the large values as being due to position calibration errors associated with the new configuration. The areal densities were taken normal to the reactor axis.

The fitted amplitudes and energies are shown in the left panel in Figure 3. The fits to the data shown as the solid lines in the plot on the right in Figure 3 are good with these approximations. For radiography at Fukushima the known concrete structure surrounding the core can be used for this calibration function.
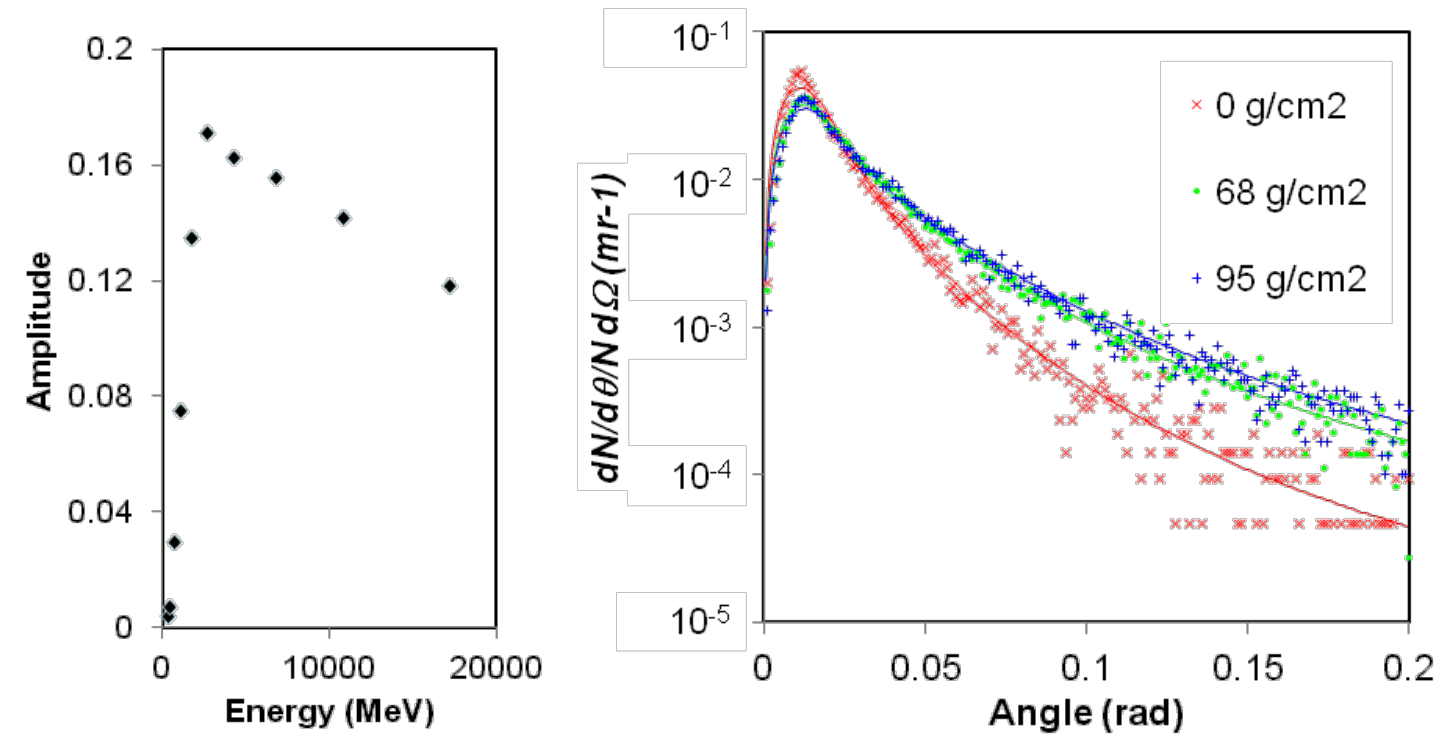

Figure 2)Left) Amplitudes and energies for the multi group fits. Right) Multi-energy group to the angular distribution data for selected regions of the data.

The images of $\mathrm{RL}$ at a plane at the center of the reactor core generated as a function of exposure time are shown in Figure 4 . The reactor core is visible after only 4 hours of exposure. After one day the void at the center can be observed. The image continues to improve through the entire 4 week experiment. 


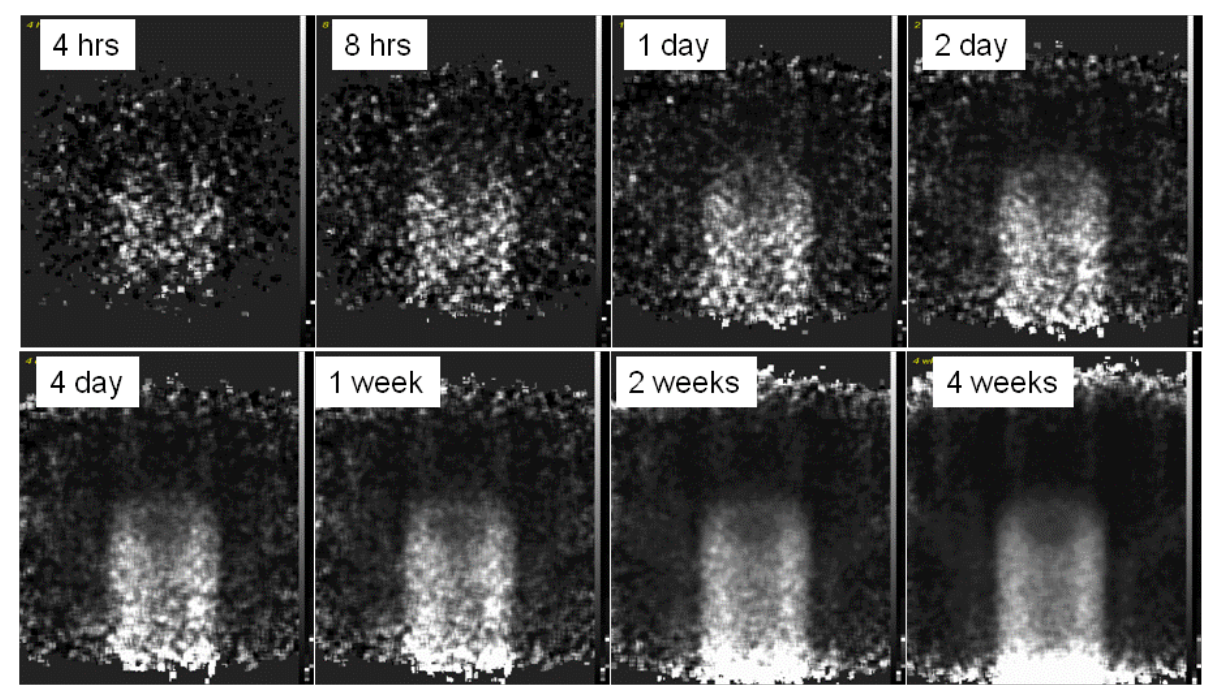

Figure 3) Time development of the radiographic signal

Images focused at different image planes along a line connecting the detector centers are shown in Figure 5. The core is best focused in the image at $200 \mathrm{~cm}$ in the detector coordinate system. At $180 \mathrm{~cm}$ the bundle of 9 fuel rods closest to the lower detector is clear, and at $220 \mathrm{~cm}$ the fuel bundle closest to the upper detector is clear. Around $250 \mathrm{~cm}$ the flange on the top of the water tank can be seen as the bottom of an oval. At $310 \mathrm{~cm}$ the steel bricks mounted in front of the upper detectors are focused. The major feature are shown in the drawing in Figure 6.

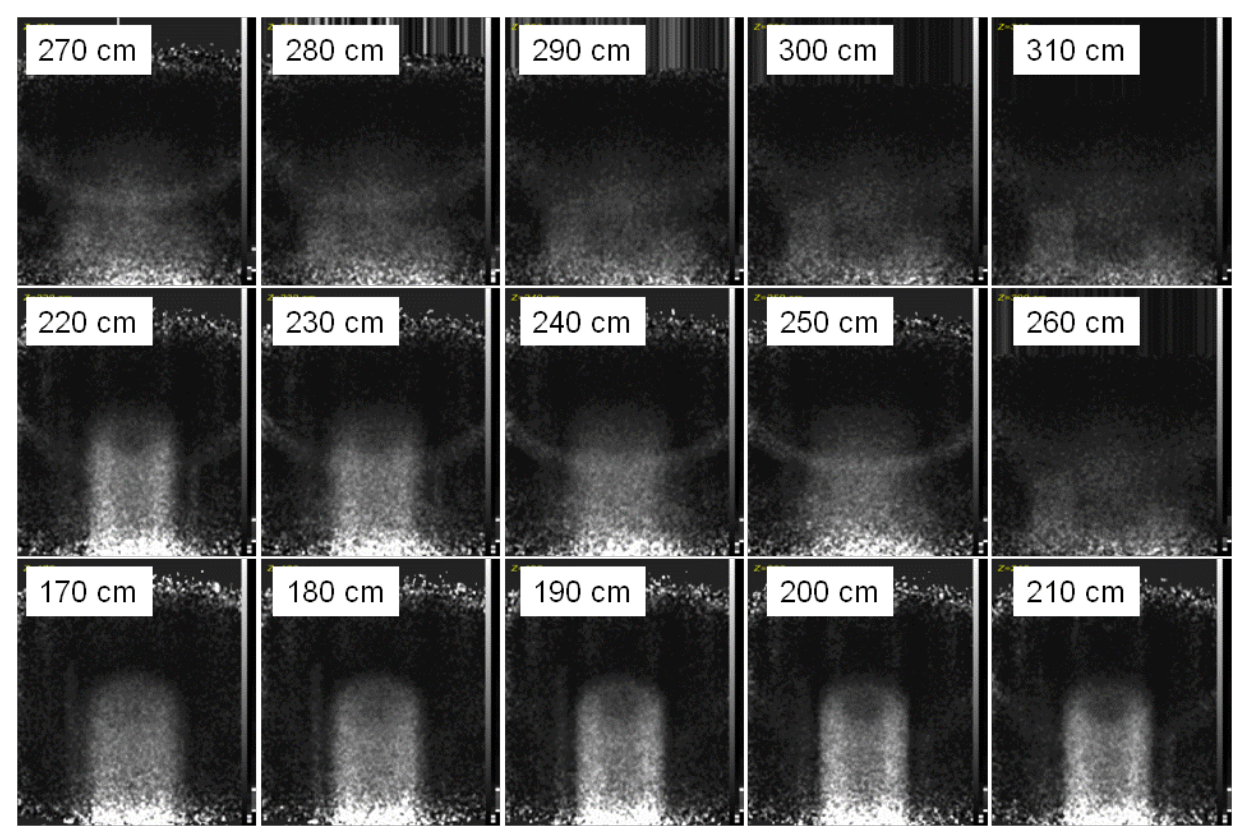

Figure 4) Slices through the tomography in $10 \mathrm{~cm}$ steps along a line connecting the detector centers. 


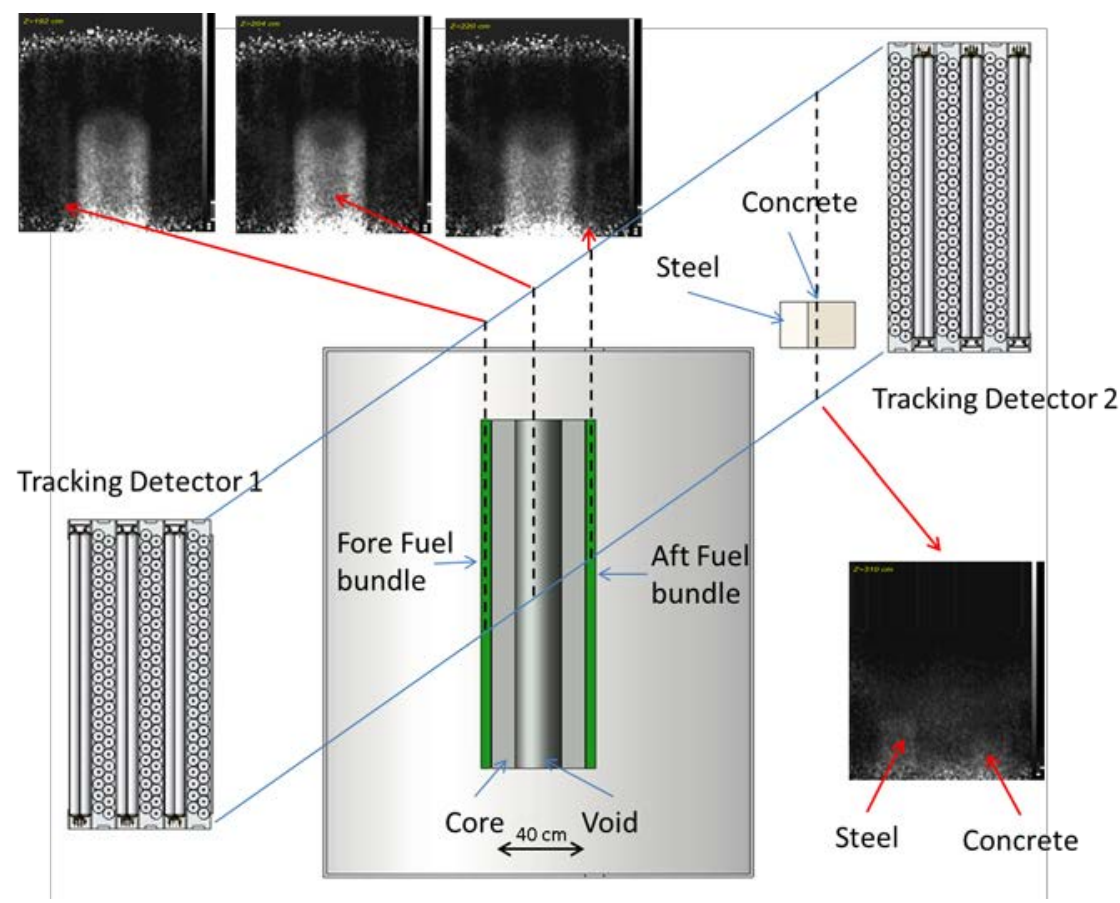

Figure 5) Some slices from the three dimension tomograph showing the major features in the NCA image.

The images have units of radiation lengths $\left(\frac{\rho_{A}}{X_{0}}\right)$. In the case of the axial symmetric core radiation length volume density can be obtained by performing an Abel inversion. Here we use a regularized Abel technique previously described in [10]. The results are shown as images and line plots in Figure 7. 

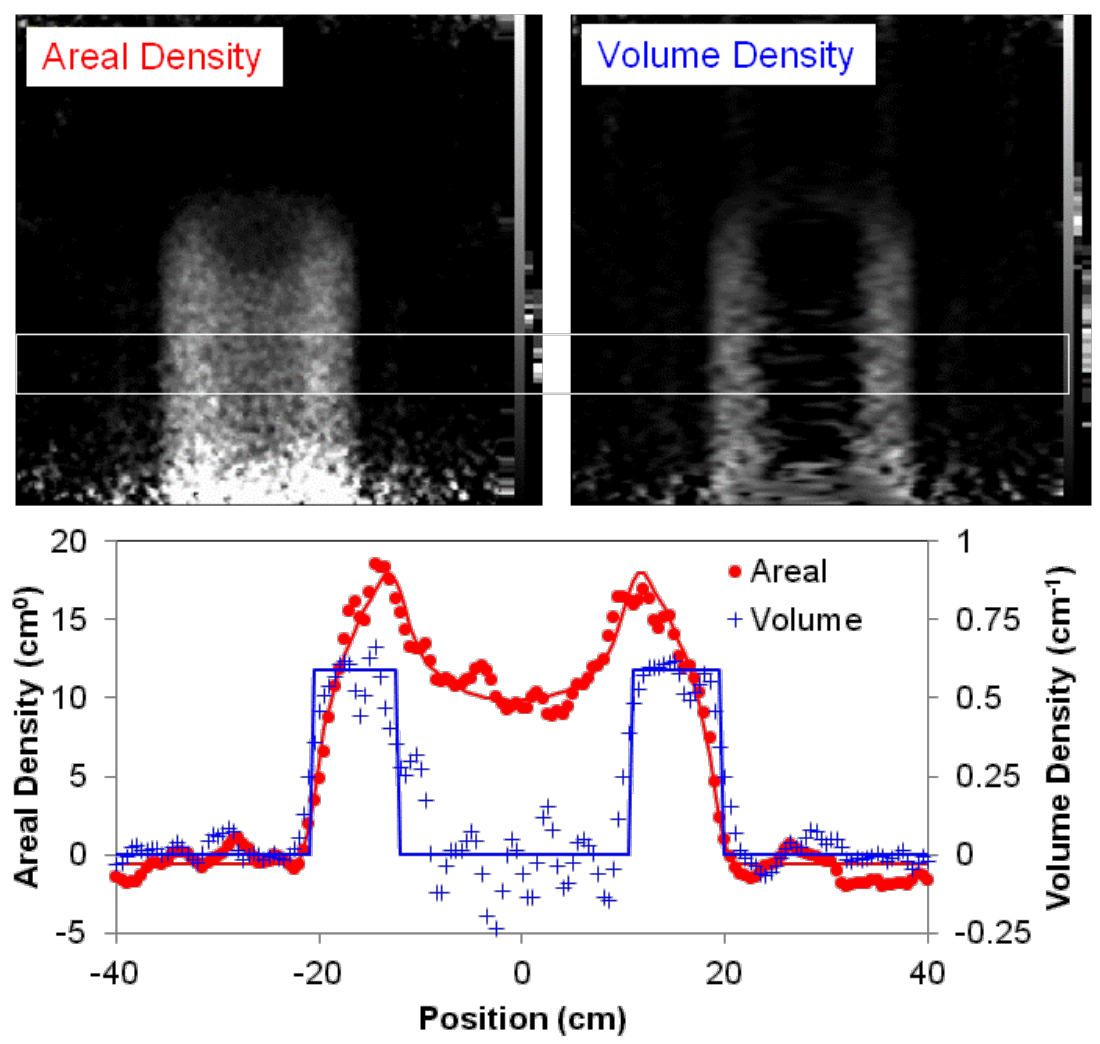

Figure 6) Top) images of the radiation length weighted areal (left) and volume (right) densities. Bottom) Line plots of the areal and volume density average over the region marked by the white lines. Data points are the experimental values and solid lines are a model fitted to the areal density data.

\section{Monte Carlo Simulations}

GEANT[2] Monte Carlo simulations of this geometry have been performed. These did not include the details of the detector or the building, so the calibration of the multi-group model was redone for the Monte Carlo data set, and the images were formed using the same reconstruction software. The $z$ values are offset by $200 \mathrm{~cm}$ because of different coordinate systems. The major features observed in the experimental data are also observed in the Monte Carlo reconstructions. 


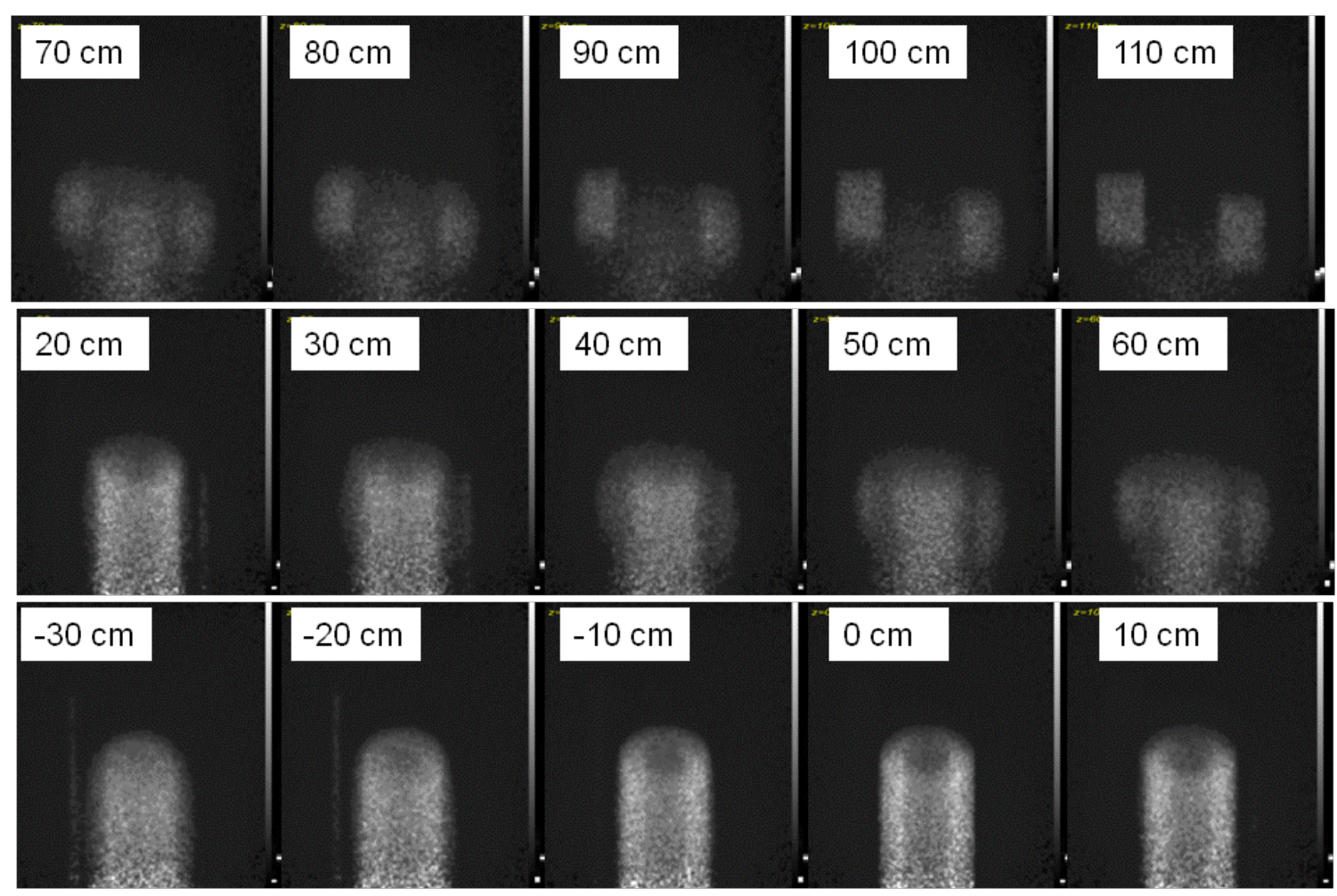

Figure 7) Slices through the tomography in $10 \mathrm{~cm}$ steps along a line connecting the detector centers. These can be compared to Figure 5.

The grey scales are the same in both sets of images. Qualitatively, the contrast from the objects is the same. The major quantitative difference is a difference on the offset (background dark level) between the two reconstructions. A comparison of the Monte Carlo results and the data is shown in Figure 9, both as horizontal density plot and images. The offsets have been adjusted to be the same. The quantitative agreement is within $3 \%$ in measured density. Similar agreement is obtained for the other slices in $\mathrm{z}$. 

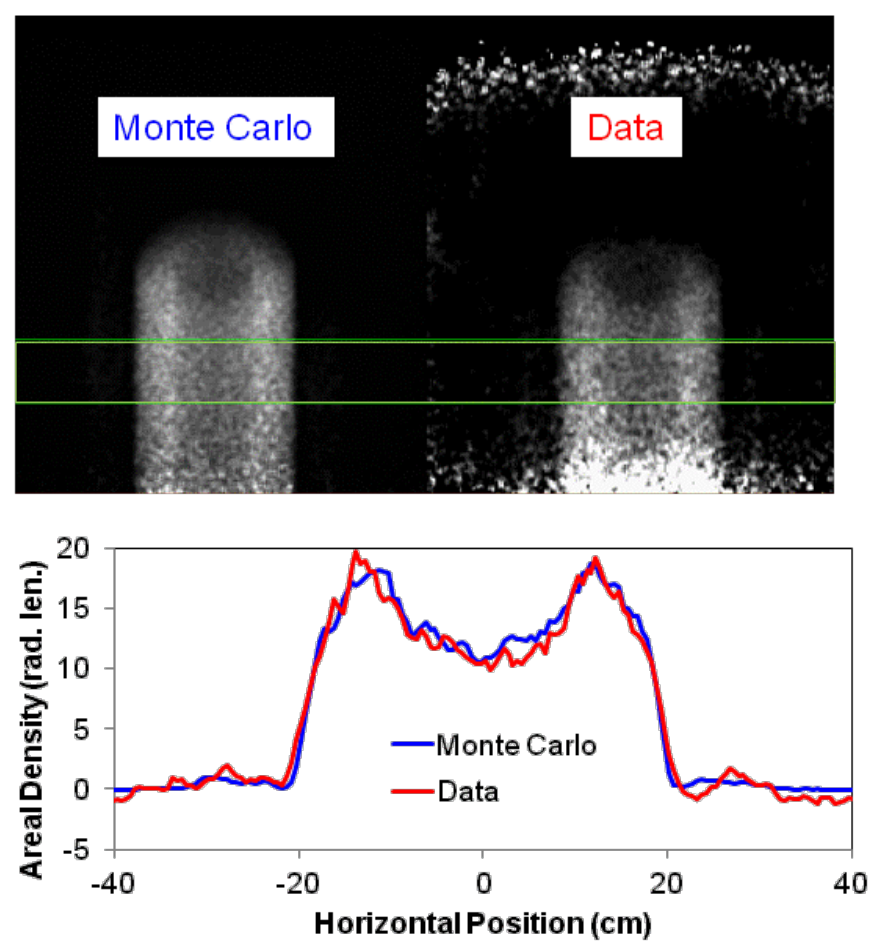

Figure 8) Comparison of reconstructed densities between the GEANT[2] Monte Carlo simulation and the data for a slice through the center of the core. Top) images with the projected region marked by the green lines. Bottom) plots of areal density vs. horizontal position for the Monte Carlo results (blue) and the data (red).

\section{Path Forward}

We have provided a report on the muon measurements at the NCA reactor using the LANL mini muon tracker. These along with our previous work[1-3] demonstrate the capabilities of scattering radiography for imaging the cores of the Fukushima Diiachi reactors. Should there be a decision to pursue muon imaging we at LANL believe that there are several steps in the path forward.

1. A practical plan for detector locations has been presented in reference [2]. Here it was assumed that detectors would be mounted on the operations floor of the turbine building to the east of Unit 2, and near the outside to the west of the reactor building the reactor building (each of these is referred to as a supermodule). Background measurements have been performed in both locations in 2012. Assuming the measured levels, which were $\sim 1 \mathrm{mSv}$ in the west locations and $0.02 \mathrm{mSv}$ in the east location, lead to the conclusion that although the east detectors can be operated with no shielding the west detectors will need to be shielded. Successful operation with the current technology will require a shielding factor of $\sim 50$. The reactor build may provide one wall of the shield if it can be decontaminated to a sufficiently low level. The design and plan for constructing this shielding is the major technical task for mounting muon imaging.

2. The tracking is performed using drift tube detectors. These consist of a small diameter wire ( $\sim 30$ $\square \mathrm{m}$ diameter) stretched to of order $60 \mathrm{~g}$ of tension at the center of a $50 \mathrm{~mm}$ diameteraluminum tube. The wires ar placed at a voltage of $\sim 2600 \mathrm{~V}$ with respect to the aluminum tube. Electrons produced in 
the gas drift at an approximately constant velocity to the wire. In the vicinity of the wire the electric field is large enough to accelerate the elections to an energy above the ionization potential of the gas and produce noise free amplification within the gas. The charge signal produce by this process is amplified and two relative times measured with two different discriminator levels are recorded in FPGA. These are packaged as a data packet that is communicated to a processor via Ethernet. The detector hits are assembled into groups of hits, called an event. The wire location and times from a group of hits are fitted by the position, angle and time zero, to form a track, and the tracts that are detected in two separated detectors and analyzed to image intervening material. Suitable detectors and electrons will need to be acquired.

3. The distance the detector poses some difficulty for the existing electronics and Ethernet architecture because of the hardwire connection needed to synchronize the clocks between the east and west supermodules. A solution to this problem is to operate the detectors independently with independent processors and assemble coincidences after the track fitting. Events could be correlated between the two supermodules using asynchronous supermodule clocks using running time corrections and tracking information. This would reduce the bandwidth requirements external to the detector assemblies. This architecture (or other solutions) could be developed and tested using the LANL MMT detectors.

4. With adequate shielding the detectors will be operated in a few $1 / 100$ s od a mSv radiation field. It is important to verify ahead of time there will be no radiation ageing issues with the detector design that is chosen for the tracking. Because the baseline design is the LANL/DSC driftube modules, these should be tested in a radiation field of several 1/10's mSv to verify longevity. Such a test could be performed using the test detectors used to measure the backgrounds at Fukushima Diachi in 2012.

Solutions to the above list of problems would ensure successful measurements of the reactor cores at Fukushima. Los Alamos would be happy to work with our Japanese partners on the items above or any others that might come up.

\section{Conclusions}

We have presented an analysis of muon radiography of the Toshiba NCA reactor. Data were taken for four weeks using the Los Alamos Mini Muon Tracker. All of the featured designed into the scene are observed in the three dimension reconstruction of the data. In addition, the data demonstrate that absolute densities can be obtained from cosmic muon radiography. Monte Carlo simulations and the data agree to within $3 \%$.

\section{References}

1. Borozdin, K., et al., Cosmic ray radiography of the damaged cores of the fukushima reactors. Physical review letters, 2012. 109(15): p. 152501.

2. Allison, J., et al., Geant4 developments and applications. Nuclear Science, IEEE Transactions on, 2006. 53(1): p. 270-278. 
3. Morris, C., et al., Charged particle radiography. Reports on progress in physics. Physical Society (Great Britain), 2013. 76(4): p. 046301-046301.

4. Bethe, H., Theory of passage of swift corpuscular rays through matter. Annalen der Physik, 1930. 5(3): p. 325-400.

5. Bethe, H.A., MOLIERE THEORY OF MULTIPLE SCATTERING. Physical Review, 1953. 89(6): p. 1256.

6. Highland, V.L., Some practical remarks on multiple scattering. Nuclear Instruments and Methods, 1975. 129(2): p. 497.

7. Lynch, G.R. and O.I. Dahl, Approximations to multiple Coulomb scattering. Nuclear Instruments \& Methods in Physics Research, Section B (Beam Interactions with Materials and Atoms), 1991. B58(1): p. 6.

8. Nakamura, K., et al., Review of Particle Physics. Journal of Physics G: Nuclear and Particle Physics, 2010. 37(7A): p. 075021.

9. Miyadera, H., et al., Imaging Fukushima Daiichi reactors with muons. AlP Advances, 2013. 3(5): p. 052133-052133-7.

10. Perry, J., et al., Imaging a nuclear reactor using cosmic ray muons. Journal of Applied Physics, 2013. 113(18): p. 184909-184909-9.

11. Morris, C.L., et al., Tomographic imaging with cosmic ray muons. Science and Global Security, 2008. 16(1-2): p. 37-53.

12. Morris, C.L., et al., Obtaining material identification with cosmic ray radiography. Aip Advances, 2012. 2(4). 


\title{
Cosmic Ray Radiography of the Damaged Cores of the Fukushima Reactors
}

\author{
Konstantin Borozdin, ${ }^{1}$ Steven Greene, ${ }^{1}$ Zarija Lukić, ${ }^{2}$ Edward Milner, ${ }^{1}$ Haruo Miyadera, ${ }^{1}$ \\ Christopher Morris, ${ }^{1, *}$ and John Perry ${ }^{1}$ \\ ${ }^{1}$ Los Alamos National Laboratory, Los Alamos, New Mexico 87544, USA \\ ${ }^{2}$ Computational Cosmology Center, Lawrence Berkeley National Laboratory, Berkeley, California 94720, USA
}

(Received 9 August 2012; published 11 October 2012)

The passage of muons through matter is dominated by the Coulomb interaction with electrons and nuclei. The interaction with the electrons leads to continuous energy loss and stopping of the muons. The interaction with nuclei leads to angle "diffusion." Two muon-imaging methods that use flux attenuation and multiple Coulomb scattering of cosmic-ray muons are being studied as tools for diagnosing the damaged cores of the Fukushima reactors. Here, we compare these two methods. We conclude that the scattering method can provide detailed information about the core. Attenuation has low contrast and little sensitivity to the core.

DOI: 10.1103/PhysRevLett.109.152501

PACS numbers: 28.41.Te, 87.59.bf, 96.50.S-

Shortly after the earthquake, tsunami, and core melt downs at the reactors in Fukushima Japan in March, 2011, several groups in both the United States and Japan realized that cosmic-ray radiography might be able to provide information about the damaged cores. Two methods of radiography using cosmic rays have been described in the past, attenuation [1-3] and scattering [4-6]. Since deploying either of these methods to study the damaged cores of the Fukushima reactors involves a major human investment because of the high radiation fields surrounding the reactors, it is important to carefully evaluate the utility of the information that can be obtained from these technologies. In this Letter, we present a comparison of imaging using these two different techniques in a common geometry using the Monte Carlo particle transport code GEANT4.

The simulation code GEANT4 [7] was used to track cosmic rays through a model of a boiling water reactor similar to Fukushima Daiichi Reactor \#1. The model of the reactor included all major structures, the reactor building, the containment vessel, and the pressure vessel. Calculations were performed for an intact core, a core with a $1 \mathrm{~m}$ diameter of material removed from the core and placed in the bottom of the pressure vessel, and no core. A schematic view of the detector placement is shown in Fig. 1. The placement of detectors outside of the reactor buildings is dictated by very high radiation levels and very limited access to the insides of the buildings.

Several approximations were made to simplify the calculation: structures outside of the field encompassed by the detectors were not included (mainly the turbine buildings);

Published by the American Physical Society under the terms of the Creative Commons Attribution 3.0 License. Further distribution of this work must maintain attribution to the author(s) and the published article's title, journal citation, and DOI. the detectors were assumed to measure position and angles perfectly; there was no gamma shielding added around the detectors; and the energy spectrum was assumed to be independent of zenith angle and was taken from the $75^{\circ}$ zenith angle measurements of Jokisch et al. [8], which corresponds to the angle of reactor core from the lower detector. A comparison of the spectra given by Jokisch et al. and by Tsuji et al. [9] shows a 50\% discrepancy at low momentum and differences in the slope at higher momenta (Fig. 2). This is indicative of the uncertainty in the normalization of our results.

The output saved from the GEANT4 runs included the input and output vectors, $\vec{X}_{\text {in }}$ and $\vec{X}_{\text {out }}$, for each incident particle. The incident flux projected to the reactor core location was used to normalize the transmission radiography (attenuation method).

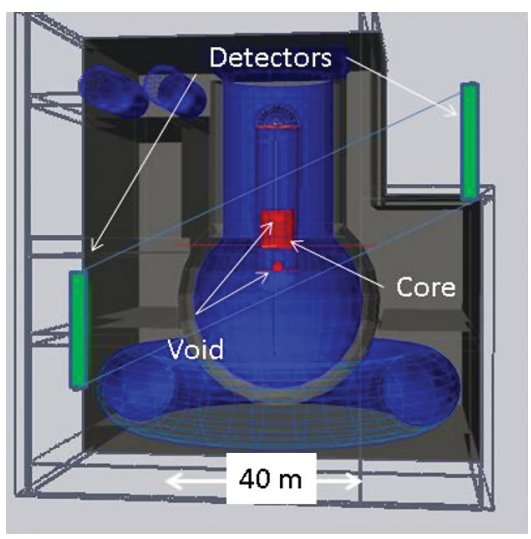

FIG. 1 (color). Cutaway view of a boiling water reactor and a schematic of the detector placement for the Monte Carlo calculation. In the case of attenuation radiography, only trajectory information from the lower detector was used. The location of the $1 \mathrm{~m}$ diameter void in the core and its placement in the bottom of the pressure vessel are indicated by arrows. 


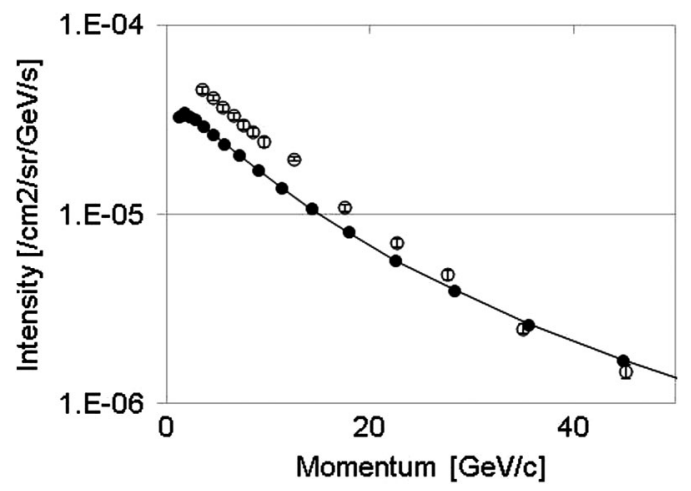

FIG. 2. Cosmic-ray muon energy spectrum at sea level. Solid symbols are from Jokisch et al. [8], and the open symbols are from Tsuji et al. [9]. Muons which penetrate the reactor lose 5-6 GeV.

The calculations are normalized to the expected $75^{\circ}$ zenith angle flux. The muon angular distribution can be approximated by [10]

$$
\begin{aligned}
\frac{d N}{d \Omega} & =\frac{3}{\pi} \cos (\theta)^{2} \text { muons } / \mathrm{min} / \mathrm{sr} / \mathrm{cm}^{2}, \\
\Omega & =\frac{\sin (\theta) h w}{l^{2}} \mathrm{sr}, \quad N=\frac{d N}{d \Omega} \Omega \sin (\theta) h w .
\end{aligned}
$$

The normalization of the angular distribution gives a total muon flux of $1 / \mathrm{cm}^{2} / \mathrm{min}$, when it is integrated over $2 \pi$ steradians. The $\sin (\theta)$ accounts for the fact that the detectors are not normal to the line that connects their centers. The modeled detectors have $h=10 \mathrm{~m}, w=5 \mathrm{~m}$, and $l=45 \mathrm{~m}$ and are mounted at $\theta=75 \mathrm{deg}$. For these conditions, we expect 5.3(2.5) $\times 10^{5}$ muons per day.

Algorithms were developed to construct images of the core using both the attenuation and multiple scattering of the cosmic rays. The goal is to determine the sensitivity of these techniques for measuring the amount of melted fuel remaining in the reactor core as well as the location of debris.

Transmission images were constructed by projecting the outgoing trajectories to a vertical plane centered in the core and histrogramming the number of events in $10 \times 10 \mathrm{~cm}^{2}$ pixels. Then, the image was calculated as $-\ln \left[N(x, y) t_{0} / N_{0}(x, y) / t_{N}\right]$, where $N_{0}$ was the incident fluence and $N$ was the transmitted fluence in exposure times of $t_{0}$ and $t_{N}$, respectively. The histogram of incident fluence was smoothed to remove an artifact introduced by the blur of the projection of the output trajectories to the plane of the core.

Plots of both the scattering images and the transmission images are shown in Fig. 3 for different exposures starting at $1 \mathrm{~h}$, increasing by near factors of 10 up to 6 weeks. These histograms are displayed with a linear gray scale with a lower value of zero in order to make the combination of contrast and statistical fluctuations clearly visible. The times for the images are for a $50 \mathrm{~m}^{2}$ detector. For a $1 \mathrm{~m}^{2}$

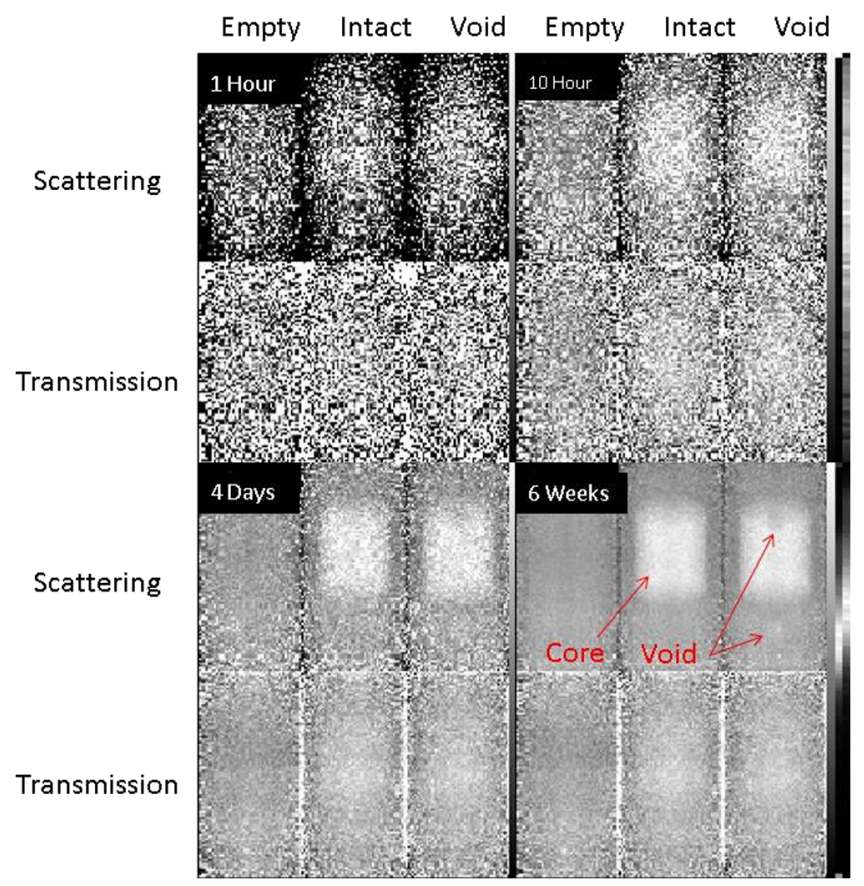

FIG. 3 (color online). Reactor reconstructions at different exposure times. In scattering radiography, the reactor core can be detected after about 10 hours of exposure. After four days, a $1 \mathrm{~m}$ diameter $(1 \%)$ void can be detected when compared to an intact core. After 6 weeks, the void is clear and the missing material can be observed. With the attenuation method, the core can be observed when compared to an empty scene in four days. The void is undetectable even after 6 weeks of exposure.

detector, these need to be increased by a factor of 50 to obtain the statistics shown at the center of the pictures. The acceptance of this geometry falls to zero at the detector edges.

At $1 \mathrm{~h}$, the difference in scattering between the images with and without the core is visible, and by ten hours the reactor core is visible in the scattering image. At 4 days, the $1 \mathrm{~m}$ diameter void is visible in the core, and by 6 weeks both the void and the resulting sphere of core material below the core are clearly visible.

The low contrast in the attenuation images is apparent when they are compared to the scattering images. The core can be detected by comparing the empty and intact images at the longer exposures, but structure in the images due to the building components shows up as strongly as the core. The void and sphere of material, clearly visible in the scattering radiograph, is not detectable in the attenuation image.

A major engineering challenge at Fukushima Daiichi is radiation shielding of deployed detectors. The site has high radiation levels on the order of $1 \mathrm{mSv} / \mathrm{h}$ dominantly produced by $\gamma$ rays from ${ }^{134} \mathrm{Cs}$ and ${ }^{137} \mathrm{Cs}$. These increase the singles counting rates and produce accidental coincidences in tracking detectors. Tests performed at the reactor site and measurements with small scale drift tube detectors 
have shown that $50 \mathrm{~cm}$ of concrete will provide adequate shielding for operating detectors at the locations modeled here. A radiation shield of precast concrete can enable quick installation to the site.

We have used GEANT4 to model cosmic-ray radiography of the Fukushima reactors. We have shown that 6 weeks (300 $\mathrm{m}^{2}$ weeks of exposure) of data provide an image with enough quality to observed $1 \%$ (a $1 \mathrm{~m}$ diameter sphere) of core material moved to a location below the core using scattering radiography. On the other hand, the same exposure in attenuation radiography shows far less sensitivity. This analysis shows that high quality data for radiography of the Fukushima cores from outside of the buildings can be accomplished with scattering radiography and large detectors. On site tests at Fukushima Daiichi have shown these measurements to be practical.

This work has been supported by the Laboratory Directed Research and Development Program of the Los Alamos National Laboratory. We would like to thank Kanetada Nagamine for valuable discussions and Christopher Fendel for his encouragement and support.

*cmorris@lanl.gov.

[1] E.P. George, Commonwealth Engineer 455 (1955).

[2] L. W. Alvarez, J. A. Anderson, F. Elbedwe, J. Burkhard, A. Fakhry, A. Girgis, A. Goneid, F. Hassan, D. Iverson, G.
Lynch, Z. Miligy, A.H. Moussa, Mohammed-Sharkawi, and L. Yazolino, Science 167, 832 (1970).

[3] K. Nagamine, M. Iwasaki, K. Shimomura, and K. Ishida, Nucl. Instrum. Methods Phys. Res., Sect. A 356, 585 (1995).

[4] K. N. Borozdin, G.E. Hogan, C. Morris, W.C. Priedhorsky, A. Saunders, L. J. Schultz, and M.E. Teasdale, Nature (London) 422, 277 (2003).

[5] C.L. Morris, C.C. Alexander, J.D. Bacon, K. N. Borozdin, D. J. Clark, R. Chartrand, C. J. Espinoza, A. M. Fraser, M. C. Galassi, J. A. Green, J. S. Gonzales, J. J. Gomez, N. W. Hengartner, G. E. Hogan, A. V. Klimenko, M. F. Makela, P. McGaughey, J. J. Medina, F.E. Pazuchanics, W.C. Priedhorsky, J.C. Ramsey, A. Saunders, R. C. Schirato, L. J. Schultz, M. J. Sossong, and G.S. Blanpied, Science and global security : the technical basis for arms control and environmental policy initiatives 16, 37 (2008).

[6] L. J. Schultz, K. N. Borozdin, J. J. Gomez, G. E. Hogan, J. A. McGill, C.L. Morris, W. C. Priedhorsky, A. Saunders, and M.E. Teasdale, Nucl. Instrum. Methods Phys. Res., Sect. A 519, 687 (2004).

[7] J. Allison, K. Amako, J. Apostolakis, H. Araujo, P. A. Dubois, M. Asai, G. Barrand, R. Capra, S. Chauvie, and R. Chytracek, IEEE Trans. Nucl. Sci. 53, 270 (2006).

[8] H. Jokisch, K. Carstensen, W. D. Dau, H. J. Meyer, and O. C. Allkofer, Phys. Rev. D 19, 1368 (1979).

[9] S. Tsuji, T. Katayama, K. Okei, T. Wada, I. Yamamoto, and Y. Yamashita, J. Phys. G 24, 1805 (1998).

[10] K. Nakamura (Particle Data Group), J. Phys. G 37, 075021 (2010). 


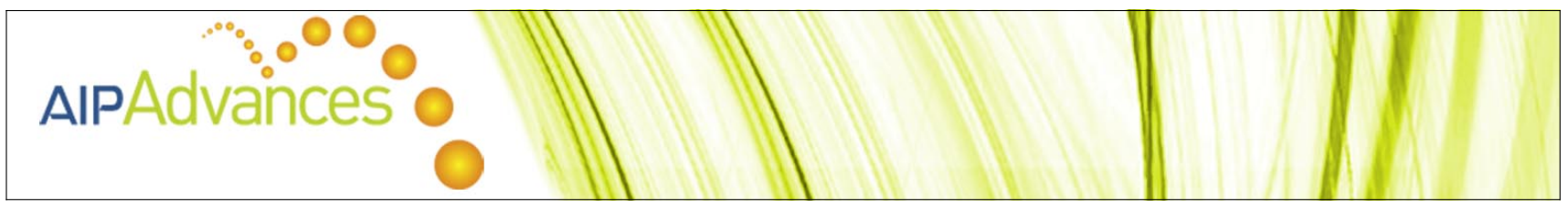

\section{Imaging Fukushima Daiichi reactors with muons}

Haruo Miyadera, Konstantin N. Borozdin, Steve J. Greene, Zarija Lukić, Koji Masuda et al.

Citation: AIP Advances 3, 052133 (2013); doi: 10.1063/1.4808210

View online: http://dx.doi.org/10.1063/1.4808210

View Table of Contents: http://aipadvances.aip.org/resource/1/AAIDBI/v3/i5

Published by the AIP Publishing LLC.

\section{Additional information on AIP Advances}

Journal Homepage: http://aipadvances.aip.org

Journal Information: http://aipadvances.aip.org/about/journal

Top downloads: http://aipadvances.aip.org/features/most_downloaded

Information for Authors: http://aipadvances.aip.org/authors

\section{ADVERTISEMENT}

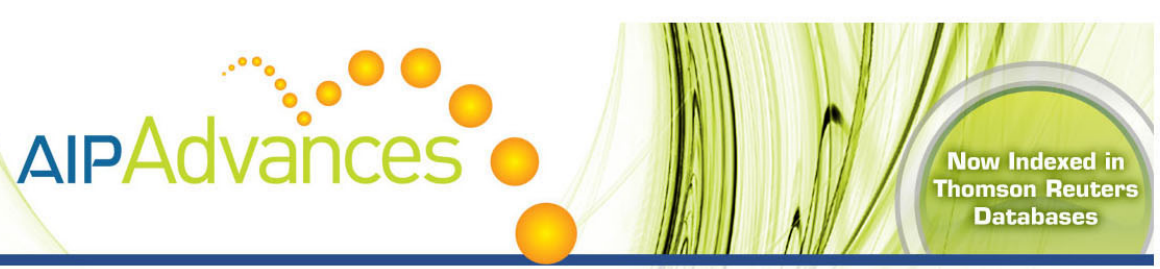

\section{Explore AIP's open access journal: •Rapid publication \\ - Article-level metrics \\ - Post-publication rating and commenting}




\title{
Imaging Fukushima Daiichi reactors with muons
}

\author{
Haruo Miyadera, ${ }^{1, a}$ Konstantin N. Borozdin, ${ }^{1}$ Steve J. Greene, ${ }^{1}$ \\ Zarija Lukić, ${ }^{2}$ Koji Masuda, ${ }^{3}$ Edward C. Milner, ${ }^{1}$ Christopher L. Morris, ${ }^{1}$ \\ and John O. Perry 1,3 \\ ${ }^{1}$ Los Alamos National Laboratory, Los Alamos, NM 87545, USA \\ ${ }^{2}$ Computational Cosmology Center, Lawrence Berkeley National Laboratory, Berkeley, \\ CA 94720, USA \\ ${ }^{3}$ University of New Mexico, Albuquerque, NM 87131, USA
}

(Received 11 February 2013; accepted 17 May 2013; published online 24 May 2013)

\begin{abstract}
A study of imaging the Fukushima Daiichi reactors with cosmic-ray muons to assess the damage to the reactors is presented. Muon scattering imaging has high sensitivity for detecting uranium fuel and debris even through thick concrete walls and a reactor pressure vessel. Technical demonstrations using a reactor mockup, detector radiation test at Fukushima Daiichi, and simulation studies have been carried out. These studies establish feasibility for the reactor imaging. A few months of measurement will reveal the spatial distribution of the reactor fuel. The muon scattering technique would be the best and probably the only way for Fukushima Daiichi to make this determination in the near future. (c) 2013 Author(s). All article content, except where otherwise noted, is licensed under a Creative Commons Attribution 3.0 Unported License. [http://dx.doi.org/10.1063/1.4808210]
\end{abstract}

The 9.0-magnitude earthquake followed by the vast tsunami on March 11, 2011, caused a nuclear crisis at Fukushima Daiichi. ${ }^{1}$ Damage of the reactor cores has attracted worldwide attention to the issue of the fundamental safety of atomic energy. ${ }^{2}$ A cold shutdown was announced by the Japanese government in December, 2011, and a new phase of cleanup and decommissioning was started. However, it is hard to plan the dismantling of the reactors without any realistic estimate of the extent of the damage to the cores, and knowledge of the location of the melted fuel. ${ }^{3,4}$ In the case of Three Mile Island, it took more than 3 years before a camera could be put into the reactor, and about 10 years before the actual damage to the reactor could be assessed. ${ }^{5}$ Since access to the reactor buildings is very limited due to high radiation fields, imaging the reactor cores from outside the buildings will be a valuable step, and can reduce the time required to dismantle the reactors significantly, resulting in cost savings and lower total worker radiation dose.

One technique for imaging the cores without access is muon imaging, which utilizes naturally occurring cosmic-ray muons to image large-scale objects. Cosmic-ray muons which have a sealevel flux of $10^{4} \mathrm{~m}^{-2} \mathrm{~min}^{-16}$ are the results of hadronic showers high in the atmosphere. Since 1950s, imaging objects by measuring transmitted muons with a muon telescope has been applied to study mine overburden, ${ }^{7}$ an Egyptian pyramid, ${ }^{8}$ a temple gate, ${ }^{9}$ volcanoes, ${ }^{10-13}$ a blast furnace ${ }^{14}$ and caverns. ${ }^{15}$ By measuring the attenuation of the muon flux, two-dimensional density maps are obtained. An approximation of muon attenuation in matter is given by:

$$
-\frac{N}{\lambda}=\frac{d N}{d x}=\frac{d N}{d E} \frac{d E}{d x} .
$$

Here, $N$ is the number of muons, $\lambda$ the attenuation length, $\mathrm{d} N / \mathrm{d} E$ the value of the muon energy spectrum at low energy, and $-\mathrm{d} E / \mathrm{d} x$ the mean energy loss rate. Since the attenuation arises by muon stop in material due to the energy loss, the transmission method is most sensitive to low atomic

ae-mail: haruo@lanl.gov 
number $(Z)$ materials where the specific energy loss is largest and the Coulomb scattering is smallest. In practical applications, muon transmission imaging often suffers from poor position resolution due to the continuous scattering along the muon path, and from low signal-to-noise ratio caused by low statistics because of small detection area (typically $\sim 2 \mathrm{~m}^{2}$ ). ${ }^{16,17}$ Also, the muon flux incident on the object is of critical importance to determine the attenuation for transmission method, which is sometimes not easy to estimate.

A more sensitive technique, muon scattering radiography, was invented at Los Alamos National Laboratory, ${ }^{18-21}$ and has been used by some other groups. ${ }^{22-24}$ The scattering method uses two muon trackers to measure incoming and outgoing tracks of individual muons, where the region of interest is contained within the acceptance of the tracker pair. Combining the incoming and outgoing tracks provides better spatial resolution when compared to the transmission method where only the information from the scattered outgoing tracks is available. The multiple Coulomb scattering ${ }^{25}$ is a stochastic process and the Gaussian width of the angle is given by: ${ }^{26}$

$$
\theta_{0}=\frac{13.6}{\beta c p} \sqrt{\frac{x}{X_{0}}}\left[1+0.038 \ln \left(\frac{x}{X_{0}}\right)\right]
$$

where $\beta c$ and $p$ are the velocity and momentum of the incident muon, and $x$ and $X_{0}$ are the thickness and radiation length of the scattering medium. The radiation lengths for water, concrete, steel and uranium are 39.3,11.6, 1.76 and $0.317 \mathrm{~cm}^{6}$. The muon momentum can be estimated by the muon scattering within the detector. ${ }^{27} \mathrm{~A}$ method for calculating most probable muon trajectory and providing higher precision in density inferences has been presented. ${ }^{28}$ Techniques used to reconstruct matter distributions are: the point of closest approach ${ }^{20,27}$ maximum-likelihood / expectation-maximization; ${ }^{29}$ Bayesian estimation. ${ }^{30}$ The scattering method has high sensitivity to high- $Z$ materials such as uranium, and is very useful for detecting them in a background of low-Z material. This method has been applied to scan trailers and shipping containers for special nuclear material. ${ }^{31,32}$ It is also a promising technique for International Atomic Energy Agency's nuclear safeguards and non-proliferation. ${ }^{33,34}$ To image Fukushima Daiichi reactors, a new analysis, displacement method, has been developed, which is described in the latter section.

A week after the Great East Japan Earthquake, we began to study applying the muon scattering technique to Fukushima Daiichi to assess damage of the reactor cores. Several groups in Japan and the US have suggested imaging the reactors with muon transmission method and compact detectors $\left(\sim 1 \mathrm{~m}^{2}\right)$. However, since uranium fuel and water give similar energy losses for muons, the fuel is difficult to distinguish from the overburden of water, concrete and steel with the transmission method. The energy loss of a muon through matter is given by:

$$
\text { Energy loss }=-d E / d x \times \text { density } \times \text { length. }
$$

The muon energy loss rate in uranium dioxide and water are 1.15 and $1.98 \mathrm{MeV} \mathrm{cm} \mathrm{g}^{-1}$ for minimally ionizing particles respectively. ${ }^{35}$ In a fuel rod, uranium dioxide pellets are packaged in a zircaloy tube, and the density of uranium fuel averaged over the active volume of the assembly is about $2.6 \mathrm{~g} / \mathrm{cm}^{3} .{ }^{36}$ Even with the whole assembly, an intact core attenuates the muon flux $\sim 2 \%$ more than water does, which makes distinguishing the reactor core from water in the presence of the overburden difficult using the transmission radiography. In contrast, the fuel gives a distinct signal in scattering radiography, producing an image contrast of $\sim 30 \%$ even through the same overburden. Also, the muon scattering and flux attenuation can be combined to distinguish materials. ${ }^{37}$ Compared to conventional transmission radiography, scattering radiography improves the spatial resolution and the image contrast by an order of magnitude for imaging reactor cores. ${ }^{38}$

In the summer of 2011, a reactor mockup was imaged using Muon Mini Tracker (MMT) at Los Alamos (altitude of 2,231 m). The MMT consists of two muon trackers each having effective detection area of $1.2 \times 1.2 \mathrm{~m}^{2}$ and consisting of 6-x and 6-y planes of sealed drift tubes. In the demonstration, cosmic-ray muons passing through a physical arrangement of material similar to a reactor were measured. The reactor mockup consisted of two layers of concrete shielding blocks with a thickness of 2.74-m each, and a lead assembly in between; one tracker was installed at 2.5-m height, and another tracker was installed on the ground level at the other side. Several arrangements of lead were studied to test specific features of the reactor imaging technique. One of the results 

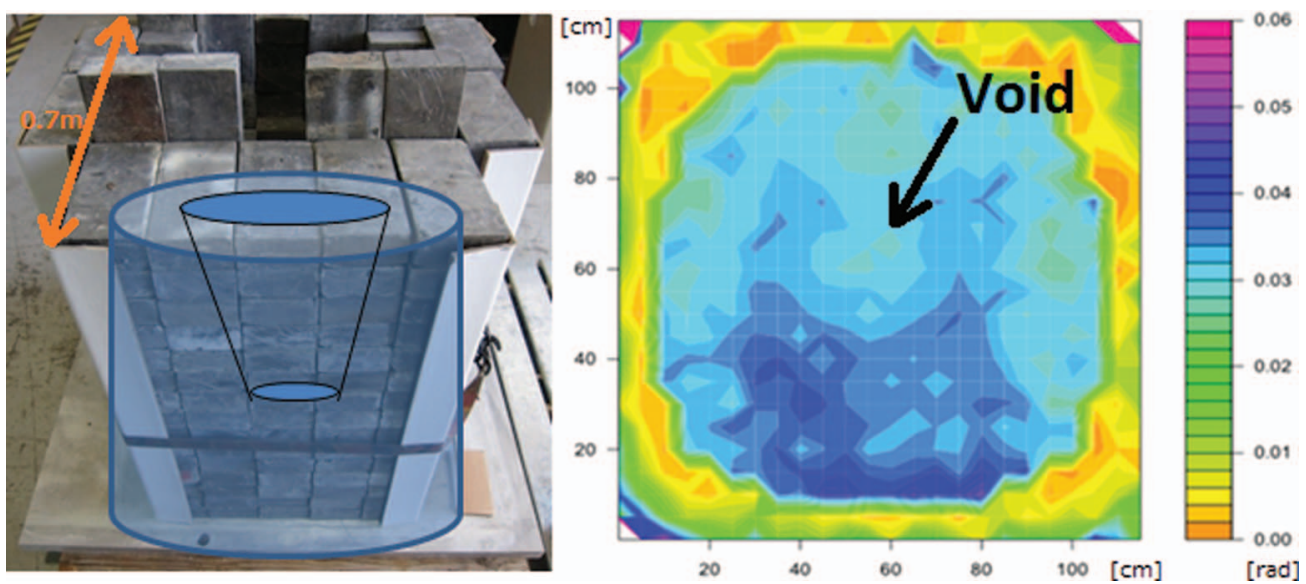

FIG. 1. Left - Lead reactor core with conic void. Right - Observed core where average scattering angles of muons are plotted. The void in the core is clearly imaged through two $2.74-\mathrm{m}$ concrete walls. The lead core of $0.7-\mathrm{m}$ thickness gives an equivalent radiation length to the uranium fuel in Unit 1, and gives a similar scattering angle. Hot spots at the corners are artifacts caused by edge effect of MMT.

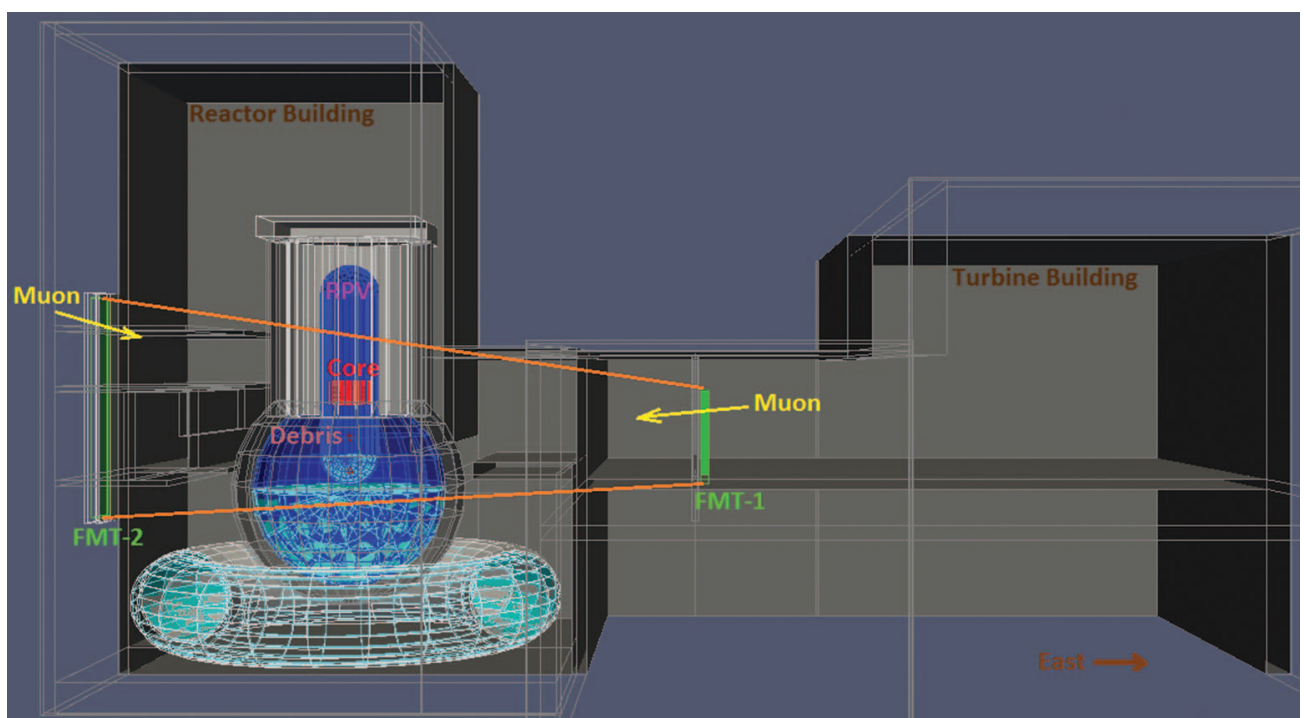

FIG. 2. Muon imaging setup for Fukushima Daiichi Unit 2. FMT-2 is installed inside a concrete radiation shield in front of the reactor building. Typical muon scattering angles are a few degrees.

is shown in Figure 1 where lead with a conical void similar in shape to the melted core of the Three Mile Island reactor was imaged through the concrete walls. It took 3 weeks to accumulate $8 \times 10^{4}$ muon events. The analysis was based on point of closest approach, where the track pairs were projected to the mid-plane of the target, and the scattered angle was plotted at the intersection. Even with event rate of an order of magnitude smaller than what we expect at Fukushima Daiichi with proposed Fukushima Muon Tracker (FMT), we successfully imaged the lead cores.

A proposed plan for Fukushima Daiichi Unit 2 is shown in Figure 2. The plan includes installation of several muon trackers on the operation floor of turbine building (FMT-1) and in front of the reactor building (FMT-2). With this geometry, muons from the east can image the bottom region of RPV while muons from the west can image the original core region. The bottom of the reactor containment vessel can be imaged by installing FMT- 2 below the ground level. Specifications of FMT- 1 and 2 are shown in Table I. They consist of gas-filled ionization drift-tube detectors made of aluminum, ${ }^{39,40}$ 
TABLE I. Specifications of FMT-1 and 2. Measurement time scales inversely with the product of total area of FMT-1 and 2 at the lowest-order approximation. However, there is a strong angular dependence of muon flux $\left(\propto \cos ^{2} \theta_{z}\right.$, where $\theta_{z}$ is the zenith angle). ${ }^{43,44}$

\begin{tabular}{lcc}
\hline \hline & FMT-1 & FMT-2 \\
\hline Detection area & $3.0 \times 3.0 \mathrm{~m}^{2}$ & $5.5 \times 5.5 \mathrm{~m}^{2}$ \\
Detector size & $3.3 \times 3.3 \times 0.6 \mathrm{~m}^{3}$ & $5.8 \times 5.8 \times 0.6 \mathrm{~m}^{3}$ \\
Drift tube & \multicolumn{2}{c}{5 -cm diameter, 12 layers (xx-yy...) } \\
Modules to be installed & $3-15$ & $2-4$ \\
\hline \hline
\end{tabular}

and have spatial and angular resolutions of $0.4 \mathrm{~mm}$ and $2 \mathrm{mrad}$ (full width at half maximum) with tracking efficiency of close to $100 \%$. The FMT system can measure muon scattering and flux attenuation simultaneously. Muon trackers of the similar sizes have been manufactured in the past with sealed drift tubes, ${ }^{41,42}$ and the technique is mature. Advantages of the drift tube compared to other muon detectors are: less $\gamma$-ray sensitivity when compared to solid state detectors $e$.g. plastic scintillators; adequate spatial resolution; mechanical robustness; operational independence of tubes resulting in negligible inefficiencies of the muon tracker in case of failures; relatively low cost. Drift-tube pulses are amplified, discriminated and digitized at the detectors in field-programmable gate-array (FPGA) time-to-digital converters (TDCs). The data are transferred to data-acquisition computers on the Fukushima Daiichi site through a dedicated Ethernet link, converted into muon tracks for online analysis, and then further analyzed in detail. The system is triggerless in the sense that tracks are built after the data have been digitized. The position calibration will be carried out in situ using the muon track data (auto calibration).

A major engineering challenge at Fukushima Daiichi is operation of the FMT in a high radiation environment. The site has radiation level of up to a few $\mathrm{mSv} / \mathrm{h}$ near the reactor buildings of Units $1-3$, which mostly consists of $\gamma$ rays from ${ }^{134} \mathrm{Cs}$ and ${ }^{137} \mathrm{Cs}$. The $\gamma$ rays trigger drift tubes through Compton scattering which mostly takes place at their aluminum walls. Though most $\gamma$-ray events can be discriminated by taking time coincidences between multiple drift-tube layers, average background rate of each drift tube must be kept below $20 \mathrm{kHz}$ so as not to exceed the bandwidth of the Ethernet. On May 25, 2012, background rate in the 0.3-m long drift tubes was measured at Fukushima Daiichi in collaboration with Tokyo Electric Power Company (TEPCO) to estimate the shield requirement for the FMT-2. The radiation levels of the locations were $0.6,0.7$ and $1.2 \mathrm{mSv} / \mathrm{h}$ on the ground. The measured background rates were $950 \mathrm{kHz}$ per $1 \mathrm{mSv} / \mathrm{h}$ (normalized to the $5.5-\mathrm{m}$ length of the FMT-2 detector). Assuming the radiation level at the installation point to be $1 \mathrm{mSv} / \mathrm{h}$, a concrete shield of 40- to $50-\mathrm{cm}$ thickness will be needed to reduce the $\gamma$-ray levels by a factor of $50 .{ }^{45}$ The radiation levels on the operation floor of turbine building are below $0.02 \mathrm{mSv} / \mathrm{h}$ at most locations in the case of Unit 2 (December 10, 2012), which allows FMT-1 to be operated without any radiation shield.

Simulation studies were performed with a geometry based on Fukushima Daiichi Unit 2 to test the feasibility of the proposed plan. Modeling studies on Unit 1 are described in the previous paper. ${ }^{38}$ GEANT4 framework ${ }^{46}$ was used and a cosmic-ray generator was implemented, which reproduces the correct energy spectrum of muons for different zenith angles in good agreement with known measurements. ${ }^{47}$ The reactor model included all the major structures of Unit 2 as shown in Figure 2. The core in the simulation had an average density of $4.3 \mathrm{~g} / \mathrm{cm}^{3}$ over the volume and consisted of uranium dioxide (60.5\%), zirconium (22\%), stainless steel (2\%) and water $(15.5 \%) ;{ }^{36}$ the debris has density of $8.2 \mathrm{~g} / \mathrm{cm}^{3}$ and consisted of uranium (70\%), zirconium (14\%), oxygen (13\%) and stainless steel (3\%), which is similar to nuclear debris found at the Three Mile Island accident. ${ }^{48}$ For the image reconstruction, we have devised a new method that uses the displacement between the projection of the incoming trajectory to the exit detector from the measurement point as illustrated in Figure 3. When a muon goes through an object, it tends to be scattered more at the latter part of the trajectory because the scattering width scales inversely with the muon energy. ${ }^{49}$ For the low energy part of the transmitted muon spectrum, most of the muon scattering can take place in the reactor core and in 


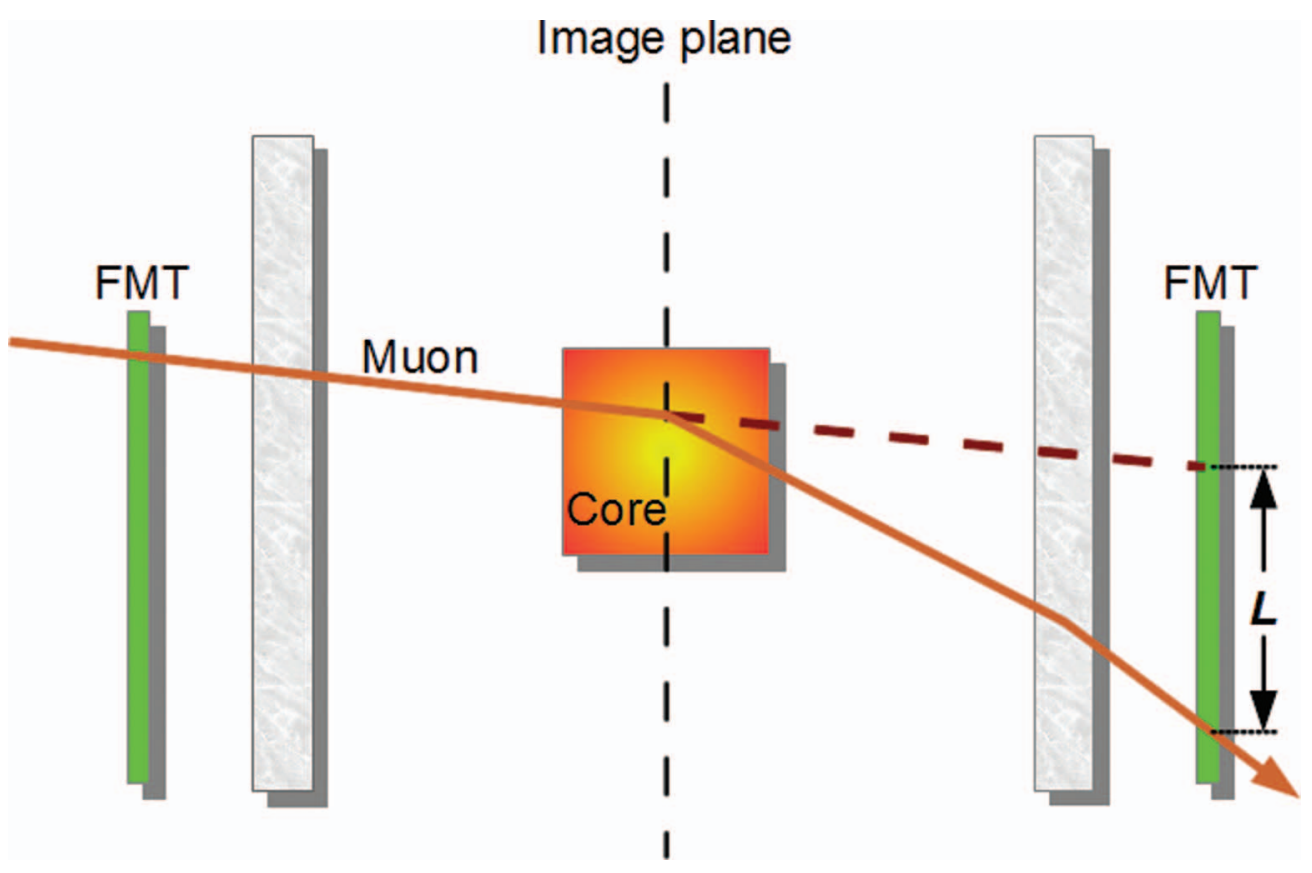

FIG. 3. Concept of image reconstruction with the displacement method. The incoming and outgoing tracks are projected to a plane at the center of the core, the intersection points are combined with weights chosen to optimize the position resolution, and a three-dimensional histogram of $x, y$, and the displacement length $(L)$ is created. The largest weight $(>80 \%)$ is placed on the incoming muon because it has higher energy and is scattered less in the intervening material between the detector and the core.

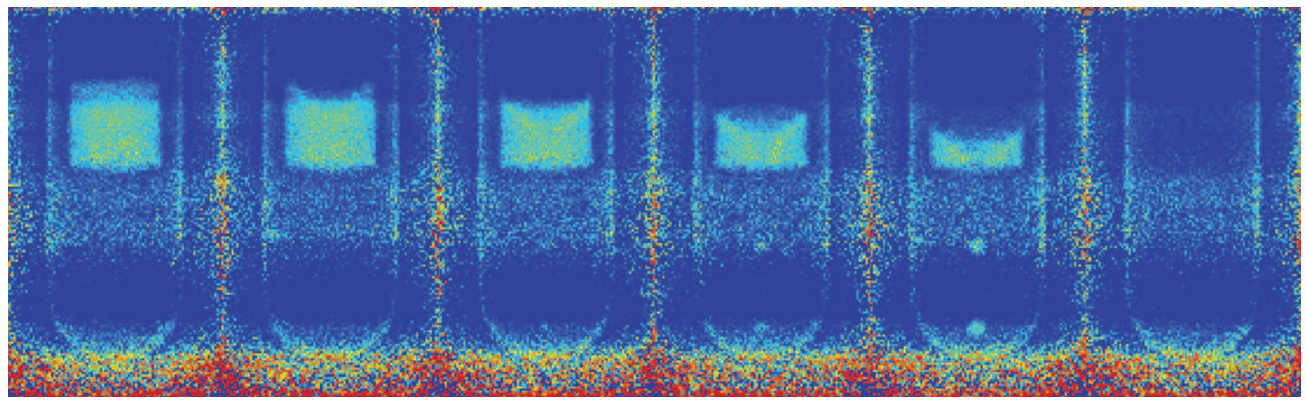

FIG. 4. Results of the GEANT4 simulations for Unit 2 with the geometry shown in Figure 2. The simulations were run with intact core (left), 10\%-, 30\%-, 50\%-, 70\%-melted core and no core (right). Two spherical debris of 10-cm (under 10\%-melted core), $20-\mathrm{cm}, 30-\mathrm{cm}$ and $40-\mathrm{cm}$ (under 70\%-melted core) radii were placed in the lower region of RPV.

the concrete after the core. Since the contribution from each scatterer scales with the distance to the exit detector $\left(L_{i}=d_{i} \theta_{i}\right)$, the latter part of the trajectory is less weighted.

The simulation results for Unit 2 with various core conditions (0 to $100 \%$ melted) analyzed with the displacement method are shown in Figure 4 where the reactor cores with conic voids were imaged through concrete walls and the steel RPV. The results correspond to 90 days of measurement with dimensions of $15 \times 9 \mathrm{~m}^{2}$ and $5.5 \times 22 \mathrm{~m}^{2}$ for FMT-1 and 2 respectively. A displacement threshold was selected to discriminate background from the water and concrete walls. Though detector resolutions are not included in the simulations, the scattered angle from the core is more than an order of magnitude larger than the detector resolution, thus they have little effect. In all cases, muon scattering is observed to provide detailed information about the reactor core allowing for quantitative assessment of the intact fraction. In addition, spherical debris of 20-, 30- and 40-cm radii can be distinguished. Figure 5 shows the image development with time (10 to 150 days) for the 


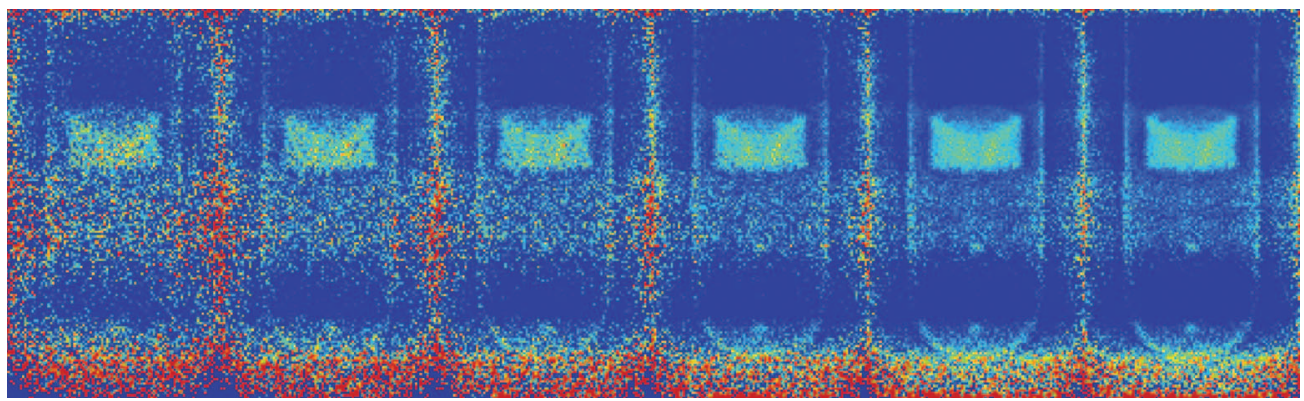

FIG. 5. GEANT4 simulations for 50\%-melted core of Unit 2 with various measurement time spans. The simulation results correspond to measurement spans of 10 (left), 20, 30, 60, 120 and 150 days (right).

$50 \%$-melted core with two debris of 20 -cm radius. The estimated event rates are $12 \mathrm{k}$ and $70 \mathrm{k}$ per day for muons that pass through both FMT-1 and 2 from the east and west sides, respectively.

As a conclusion, feasibility of assessing the damage of the Fukushima Daiichi reactors with muon scattering imaging is shown. Muons are strongly deflected by high-Z materials such as uranium, which enables the scattering technique to spot them in a reactor. A few months of measurement will reveal the distribution of the reactor-core fuel materials, and can guide planning and execution of reactor dismantlement, potentially reducing overall project span by many years.

The authors wish to thank Y. Otsuka, D. Yamada and TEPCO for suggestions and providing information. We acknowledge J.D. Bacon, L.J. Barber, M.I. Brockwell, K. Chung, M.C. Everhart, K. Nagamine, S.C. Scott, D.S. Seely, D. Tupa for help and discussions. We thank C.J. Fendel for his generous support. This work has been sponsored by the Laboratory Directed Research and Development Program of Los Alamos National Laboratory, and CC2 LLC.

${ }^{1}$ G. Brumfiel and D. Cyranoski, Nature 471, 273 (2011).

${ }^{2}$ T. N. Srinivasan and T. S. Gopi Rethinaraj, Energy Policy 52, 726 (2013).

${ }^{3}$ R. Stone, Science 25, 1507 (2011).

${ }^{4}$ P. C. Burns, R. C. Ewing, and A. Navrotsky, Science 335, 1184 (2012).

${ }^{5}$ J. S. Walker, Three Mile Island: a nuclear crisis in historical perspective (Univ. of California Press, 2004).

${ }^{6}$ J. Beringer et al., Phys. Rev. D 86, 010001 (2012).

${ }^{7}$ E. P. George, Commonwealth Engineer, 455 (July 1, 1955).

${ }^{8}$ L. W. Alvarez et al., Science 167, 832, (1970).

${ }^{9}$ S. Minato, Mater. Eval. 46, 1468 (1988).

${ }^{10}$ K. Nagamine, M. Iwasaki, K. Shimomura, and K. Ishida, Nucl. Instr. and Meth. A 356, 585 (1995).

${ }^{11}$ H. K. M. Tanaka, K. Nagamine, S. N. Nakamura, and K. Ishida, Nucl. Instr. and Meth. A 555, 164 (2005).

${ }^{12}$ J. Marteau et al., Nucl. Instr. and Meth. A 695, 23 (2012).

${ }^{13}$ Felix Fehr, J. Phys. Conf. Ser. 375, 052019, (2012).

${ }^{14}$ K. Nagamine et al., Proc. Jpn. Acad. B 81, 257 (2005).

${ }^{15}$ G. G. Barnaföldi et al., Nucl. Instr. and Meth. A 689, 60 (2012).

${ }^{16}$ N. Lesparre et al., Geophys. J. Int. 183, 1348 (2010).

${ }^{17}$ L. Malmqvist, G. Jösson, K. Kristiansson, and L. Jacobsson, Geophysics 44, 1549 (1979).

${ }^{18}$ K. N. Borozdin et al., Nature 422, 277 (2003).

${ }^{19}$ W. C. Priedhorsky et al., Rev. of Sci. Instr. 74, 4294 (2003).

${ }^{20}$ K. N. Borozdin et al., Nucl. Sci. Symp. Conf. Rec. 2003 IEEE, Vol. 2, 1061.

${ }^{21}$ G. E. Hogan et al., AIP Conf. Proc. 698, 755 (2004).

${ }^{22}$ S. Pesente et al., Nucl. Instr. and Meth. A 604, 738 (2009).

${ }^{23}$ V. Anghel et al., Nucl. Sci. Symp. and Med. Imag. Conf. 2011 IEEE, 959.

${ }^{24}$ A. A. Borisov et al., Instr. and Exp. Tech. 55, 151 (2012).

${ }^{25}$ H. A. Bethe, Phys. Rev. 89, 1256 (1953).

${ }^{26}$ G. R. Lynch and O. I. Dahl, Nucl. Instrum. Methods B 58, 6 (1991).

${ }^{27}$ L. J. Schultz et al., Nucl. Instr. and Meth. A 519, 687 (2004).

${ }^{28}$ C. J. Benton, N. D. Smith, S. J. Quillin, and C. A. Steer, Nucl. Instr. and Meth. A 693, 154 (2012).

${ }^{29}$ L. J. Schultz et al., IEEE Transactions on Image Processing 16, 1985 (2007).

${ }^{30}$ G. Wang, L. J. Schultz, and J. Qi, IEEE Transactions on Image Processing 18, 1080 (2009).

${ }^{31}$ C. L. Morris et al., Science \& Glorbal Security 16, 37 (2008).

32 "Nuclear detection system deployed at Freeport," World Cargo News (August 10, 2012).

${ }^{33}$ G. Jonkmans, V. N. P. Anghel, C. Jewett, and M. Thompson, Ann. Nucl. Energy 53, 267 (2013). 
${ }^{34}$ International Atomic Energy Agency, IAEA Technical Report Series 392, (1998).

${ }^{35}$ D. E. Groom, N. V. Mokhov, and S. I. Striganov, At. Nucl. Data Table 78, 183 (2001).

${ }^{36}$ US Department of Energy, Integrated Data Base Report 1996, DOE/RW-0006-Rev.13, 1-8.

${ }^{37}$ C. L. Morris et al., AIP Advances 2, 042128 (2012).

${ }^{38}$ K. Borozdin et al., Phys. Rev. Lett. 109, 152501 (2012).

${ }^{39}$ Z. Wang et al., Nucl. Instr. and Meth. A 605, 430 (2009).

${ }^{40}$ Z. Wang et al., Nucl. Instr. and Meth. A 703, 91 (2013).

${ }^{41}$ J. A. Green et al., IEEE Nucl. Sci. Symp. Conf. Rec. 2006 IEEE, 285.

${ }^{42}$ R. Rios et al., Nucl. Instr. and Meth. A 637, 105 (2011).

${ }^{43}$ J. Kempa and I. M. Brancus, Nucl. Phys. B (Proc. Suppl.) 122, 279 (2003).

${ }^{44}$ A. Tang, G. Horton-Smith, V. A. Kudryavtsev, and A. Tonazzo, Phys. Rev. D 74, 053007 (2006).

${ }^{45}$ A. Shimizu, T. Onda, and Y. Sakamoto, J. Nucl. Sci. and Tech. 41, 413 (2004).

${ }^{46}$ S. Agostinelli et al., Nucl. Instr. and Meth. A 506, 250 (2003).

${ }^{47}$ H. Jokisch et al., Phys. Rev. D 19, 1368 (1979).

${ }^{48}$ D. W. Akers, S. M. Jensen, and B. K. Schuetz, U.S. Nuclear Regulatory Commission, NUREG/CR-6195 (1994).

${ }^{49}$ U. Schneider and E. Pedroni, Med. Phys. 21, 1657 (1994). 